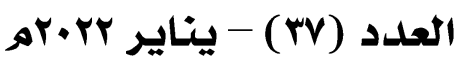

الترقيه : الدولي للنسخت الالكترونيت:
مجلت كاليت التربيت - جامعت بورسعيد

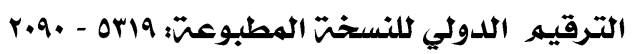

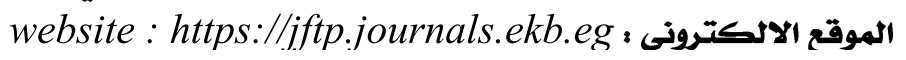

\title{
Efficacité de l'apprentissage basé sur le cerveau pour acquérir quelques règles grammaticales en français chez les étudiants du cycle secondaire
}

\author{
Prof.Dr. Sabri Eid Gad El Said \\ Professeur des Curricula \\ et Didactique du FLE \\ Faculté de Pédagogie \\ Université d'Hélouan
}

\author{
Dr. El Adl Ahmed El Mahdi \\ Maître de conférences des curricula et \\ des méthodologies du FLE \\ Faculté de Pédagogie \\ Université de Port Said
}

\section{Racha Fouad Mohamed Abdel Hamid}

Faculté de Pédagogie

Université de Port Said

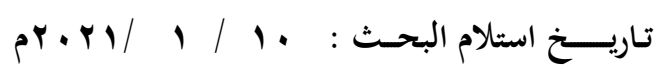

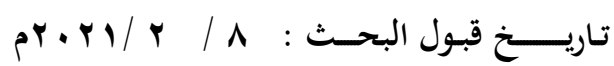

rasha.fouad@edu.psu.edu.eg: البريد الالكتروني للباحث اليحث : 
Efficacité de l'apprentissage basé sur le cerveau pour acquérir quelques règles grammaticales en français chez les étudiants du cycle secondaire

Prof.Dr. Sabri Eid Gad El Said \& Dr. El Adl Ahmed El Mahdi \& Racha Fouad Mohamed Abdel Hamid

\section{RÉSSUMÉ:}

L'étude actuelle vise à vérifier l'efficacité de l'apprentissage basé sur le cerveau pour acquérir les règles grammaticales chez les étudiants de la deuxième année secondaire aux écoles de langues. Et pour réaliser cet objectif, la chercheuse a élaboré les outils suivants: une grille des règles grammaticales que les étudiants de l'échantillon étudient au deuxième semestre et un test des règles grammaticales.

Et l'échantillon de l'étude composé de 60 élèves en deuxième année secondaire aux écoles de Fakhr Al-Dakahlia officielles de langues à Mansourah au Gouvernorat d'Al-Dakahlia qui étudient le français comme deuxième langue étrangère. Cet échantillon a réparti en deux groupes: Un groupe expérimental (un groupe de 30 étudiants qui apprend selon l'apprentissage basé sur le cerveau) et un groupe témoin (un groupe de 30 étudiants qui apprend selon la méthode traditionnelle)

Les résultats de l'étude indiquent l'efficacité de l'apprentissage basé sur le cerveau pour acquérir quelques règles grammaticales en français chez l'échantillon de l'étude.

MO'IS CLÉS : Efficacité- Apprentissage basé sur le cerveau- Règles grammaticales 
Efficacité de l'apprentissage basé sur le cerveau pour acquérir quelques règles grammaticales en français chez les étudiants du cycle secondaire

Prof.Dr. Sabri Eid Gad El Said \& Dr. El Adl Ahmed El Mahdi \& Racha Fouad Mohamed Abdel Hamid

\section{unill|}

يهاف هذا البحث الى التحقى من فعالية التعلم القائم على الاماغ فى اكتساب بعض القواعد

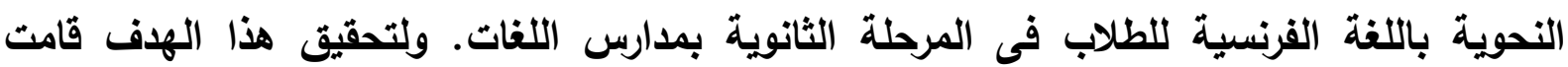

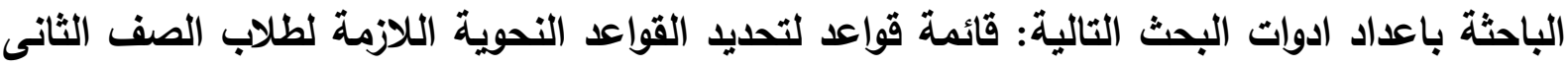
الثانوى بمدارس اللغات، واختبار القواعد النحوية. وقد شملت عينة البحث على ستون طالب وطالبة من الصف الثانى الثانوى بمدارس فخر الدقهلية الرسمية للغات فى المنصورة بمحافظة الدقهلية وقدان

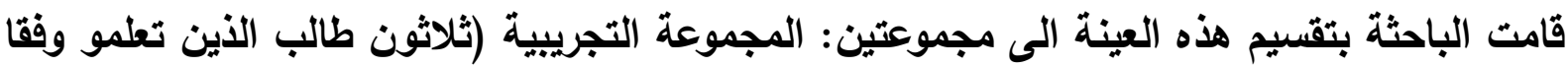
للتطلم القائم على الاماغ) والمجموعة الضابطة ( ثلاثون طالب الذين تعلمو وفقا للطريقة التقليدية). وقد اثتبت نتائج هذا البحث الى ان هناك فاعلية للتعلم القائم على الاماغ فى اكتساب القواعد النحوية

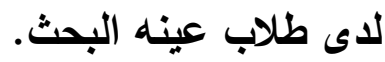
الكمات الإنتاصية فعالية- التعلم القائم على الاماغ- القواعد النحوية. 
Efficacité de l'apprentissage basé sur le cerveau pour acquérir quelques règles grammaticales en français chez les étudiants du cycle secondaire

Prof.Dr. Sabri Eid Gad El Said \& Dr. El Adl Ahmed El Mahdi \& Racha Fouad Mohamed Abdel Hamid

\section{IN'TRODUC'TION}

Il est indéniable que la langue joue un rôle si important dans la vie des individus et des sociétés. Elle permet à l'homme d'étendre son savoir et son influence à travers les siècles où il l'utilise pour se communiquer et exprimer ses besoins, ses ambitions et ses sentiments. Grâce à la langue, l'homme peut s'échanger les expériences et les connaissances avec les autres.

La langue française, comme langue étrangère vivante, elle est la langue officielle de nombreux pays. Elle est largement utilisée dans un certain nombre d'autres pays. Chaque langue étrangère comporte quatre compétences essentielles: écouter, parler, lire et écrire.

La grammaire constitue la théorie de la langue. Elle est nécessaire et indispensable dans le processus d'acquisition de la langue. En outre, les règles grammaticales sont les éléments les plus importants à la communication linguistique. Aussi, la grammaire se considère la compétence linguistique qui aide à développer les quatre compétences fondamentales pour apprendre une langue étrangère. Elle joue donc un rôle très important dans la maîtrise d'une langue.

Bien que l'importance de la grammaire dans l'enseignement/ apprentissage d'une langue étrangère, surtout la langue française, il y a une faiblesse au niveau des élèves à la grammaire, notamment aux différents cycles éducatifs d'après plusieurs études citons celles-ci: Gad (1990), Salem (1996), Nawar (2004), Ali (2007), Zamam (2012) Abou Elyazid (2013), Shehata (2013), Salem (2014).

En conséquence, on devrait utiliser les stratégies et les méthodes d'apprentissage qui aident à acquérir les règles grammaticales, l'apprentissage basé sur le cerveau est l'une de ces stratégies qui a prouvé son efficacité sur l'acquisition des règles grammaticales. Il y a beaucoup d'études qui ont démontré l'efficacité de cette stratégie en vue d'acquérir les règles grammaticales comme: Yaguello (2003), Davidson (2009), Connell (2009), Dominey et al. (2009), Ulte, et al. (2010), Pennington (2010), Varies et al. (2010), Levesque (2013). Pineau et al. (2013) assurent que la forme du cerveau joue un rôle essentiel sur la capacité d'apprentissage des apprenants. L'anatomie du cerveau influe sur le contrôle cognitif, la compétence essentielle pour l'apprentissage et la réussite scolaire.

D'autre part, Belouga (2016) montre que le cerveau joue un rôle plus important comme il comprend des millions de cellules nerveuses, ou neurones. Compte tenu de leur nombre, ces neurones peuvent être reliés par une infinité de connexions. Chaque fois que nous apprenons quelque chose, ce réseau s'enrichit de nouvelles connexions. De même, chaque fois que nous viennent de nouvelles idées, celles-ci correspondent à la formation 
Efficacité de l'apprentissage basé sur le cerveau pour acquérir quelques règles grammaticales en français chez les étudiants du cycle secondaire

Prof.Dr. Sabri Eid Gad El Said \& Dr. El Adl Ahmed El Mahdi \& Racha Fouad Mohamed Abdel Hamid

de nouvelles connexions entre des neurones. Ainsi, plus nous faisons travailler notre cerveau, plus il gagne en efficacité.

\section{PROBLÉMA'TIOUE DE LA RECHERCHE}

La problématique de la recherche actuelle réside dans la faiblesse du niveau des étudiants de la deuxième année secondaire aux écoles de langues en ce qui concerne les règles grammaticales.

Pour vaincre ce problème, la recherche actuelle essaie de répondre aux questions suivantes:

1. Jusqu' à quel niveau les élèves de l'échantillon de la recherche maîtrisentils ces règles grammaticales?

2. Quelle est l'efficacité de l'apprentissage basé sur le cerveau pour l'acquisition des règles grammaticales chez les étudiants de la deuxième année secondaire aux écoles de langues?

Objectifs de la recherche

1. Identifier le niveau actuel les étudiants de la deuxième année secondaire aux écoles de langues en ce qui concerne les règles grammaticales en français.

2. Vérifier l'efficacité de l'apprentissage basé sur le cerveau pour acquérir les règles grammaticales chez les étudiants de la deuxième année secondaire aux écoles de langues.

\section{IMPOR'TANCE DE IA RECHERCHE}

Cette recherche essaie de/d:

1. Acquérir quelques règles grammaticales chez les étudiants de la deuxième année secondaire de langues à travers la stratégie de l'apprentissage basé sur le cerveau.

2. Encourager les chercheurs en didactique du FLE à s'intéresser à la faiblesse dans les règles grammaticales et à acquérir ces règles grammaticales chez les apprenants.

3. Solliciter l'attention des orienteurs et des enseignants du FLE sur l'utilisation de la stratégie de l'apprentissage basé sur le cerveau aux différents cycles éducatifs.

4. Développer l'aptitude à fonctionner les règles grammaticales dans les différentes situations communicatives chez les étudiants de l'échantillon de la recherche.

\section{HYPO'THÈSES DE LA RECHWRCHE}

1. Il existe une différence statistiquement significative au niveau $(0.05)$ entre les moyennes des notes des étudiants du groupe expérimental et celles des étudiants du groupe témoin au post-test des règles grammaticales en faveur du groupe expérimental. 
Efficacité de l'apprentissage basé sur le cerveau pour acquérir quelques règles grammaticales en français chez les étudiants du cycle secondaire

Prof.Dr. Sabri Eid Gad El Said \& Dr. El Adl Ahmed El Mahdi \& Racha Fouad Mohamed Abdel Hamid

2. Il existe une différence statistiquement significative au niveau (0.05) entre les moyennes des notes des étudiants du groupe expérimental au pré- post test des règles grammaticales en faveur du post-test.

3. Il existe une efficacité de l'apprentissage basé sur le cerveau pour l'acquisition des règles grammaticales chez les étudiants de l'échantillon de la recherche.

\section{LIMI'LS DE IA RECHERCHE}

Cette recherche se limite à :

- Un échantillon composé de 60 étudiants en deuxième année secondaire aux écoles de Fakhr Al-Dakahlia officielles de langues à Mansourah au Gouvernorat d'Al-Dakahlia.

Outils de la recherche

1. Une grille des règles grammaticales que les étudiants de l'échantillon étudient au deuxième semestre.

2. Un test des règles grammaticales.

\section{MÉTHODOLOGIE DE IA RECHERCHE}

Cette recherche a adopté deux méthodes:

1. La méthode descriptive: En ce qui concerne les principes philosophiques de la recherche (la grammaire et l'apprentissage basé sur le cerveau).

2. La méthode quasi-expérimentale: En ce qui concerne l'application du pré /post test des règles grammaticales pour vérifier l'efficacité de l'apprentissage basé sur le cerveau pour l'acquisition des règles grammaticales chez les étudiants de la deuxième année secondaire aux écoles de langues.

\section{DÉMARCHE DE IA RECHERCHE}

1- Pour répondre à la première question, la chercheuse a procédé selon la démarche suivante:

- Préparer un pré/post test pour détecter à quel niveau les étudiants de la deuxième année secondaire aux écoles de langues maîtrisent les règles grammaticales.

- Présenter ce test aux membres du jury en didactique du FLE pour déterminer sa validité.

2- Pour répondre à la deuxième question, la chercheuse a procédé selon la démarche suivante:

- Reformuler le contenu concernant les règles grammaticales à la méthode de français que les étudiants de la deuxième année secondaire aux écoles de langues étudient au deuxième semestre selon l'apprentissage basé sur le cerveau 
Efficacité de l'apprentissage basé sur le cerveau pour acquérir quelques règles grammaticales en français chez les étudiants du cycle secondaire

Prof.Dr. Sabri Eid Gad El Said \& Dr. El Adl Ahmed El Mahdi \& Racha Fouad Mohamed Abdel Hamid

- Choisir les élèves de l'échantillon de la recherche et les répartir en deux groupes (un groupe expérimental et un groupe témoin).

- Appliquer le pré-test sur l'échantillon de la recherche.

- Enseigner les règles grammaticales à la méthode de français que les étudiants de la deuxième année secondaire aux écoles de langues étudient au deuxième semestre selon l'apprentissage basé sur le cerveau et au groupe témoin selon la méthode traditionnelle.

- Appliquer le post-test sur l'échantillon de la recherche.

- Analyser les résultats en utilisant les méthodes statistiques convenables.

- Interpréter les résultats de la recherche.

- Proposer les recommandations et les suggestions de la recherche.

\section{'TERMINOLOGIE DE IA RECHERCHE}

Apprentissage basé sur le cerveau

Selon Jensen (2000), l'apprentissage basé sur le cerveau est une approche scientifique de l'enseignement qui étudie les mécanismes neuronaux du cerveau pour faciliter l'apprentissage.

Caine et Caine (2008) définissent l'apprentissage basé sur le cerveau comme la théorie qui contient la connaissance des règles du cerveau, connaître la façon dont le cerveau apprend, ensuite l'organisation de l'enseignement selon ces règles.

Pour la chercheuse, l'apprentissage basé sur le cerveau est une stratégie d'apprentissage qui aide l'apprenant à acquérir les connaissances, à améliorer son niveau cognitif, à stimuler sa réflexion et à réaliser les tâches éducatives de manière efficace.

Règles grammaticales

D'après Fuchs et Le Goffic (2000), la grammaire est un système dont il s'agit de dégager les lois d'organisation et de fonctionnement.

Mais Larousse (2002) voit que la grammaire est une science des règles du langage parlé ou écrit. La morphologie et la syntaxe sont les deux parties principales de la grammaire. Elle est étude systématique des éléments constitutifs d'une langue".

La chercheuse définit les règles grammaticales comme un ensemble de règles à suivre pour écrire et parler correctement une langue et l'employer efficacement dans les différentes situations.

\section{CADRE 'THÉORIOUE}

\section{L'APPREN'TISSAGE BASÉ SUR LE CERVEAU}

Importance de l'apprentissage basé sur le cerveau

L'apprentissage basé sur le cerveau a une grande importance dans la création d'un environnement d'apprentissage / enseignement riche. Elle 
Efficacité de l'apprentissage basé sur le cerveau pour acquérir quelques règles grammaticales en français chez les étudiants du cycle secondaire

Prof.Dr. Sabri Eid Gad El Said \& Dr. El Adl Ahmed El Mahdi \& Racha Fouad Mohamed Abdel Hamid

joue aussi un rôle essentiel dans les moyens d'acquisition des connaissances et des expériences.

Selon CERI (2008) l'importance de l'apprentissage basé sur le cerveau se résume en:

- L'apprentissage basé sur le cerveau peut approfondir les connaissances de base sur l'apprentissage en tant qu'aspect central de la vie humaine et sociale, en transcendant les différentes institutions éducatives et le domaine éducatif.

- Il permet de révéler des particularités non apparentes, afin de proposer des moyens de remédiation - par exemple pour surmonter des troubles de l'apprentissage de la lecture, ou une dyscalculie. Ces méthodes pourraient aussi permettre d'améliorer l'apprentissage.

- La capacité à la conception des pratiques éducatives, surtout en ce qui concerne l'équilibre entre, d'un côté, la meilleure façon d'apprendre et le meilleur moment pour apprendre.

- Il permet d'avancer de la meilleure façon d'enseigner différencié.

- Il aide à renforcer l'apprentissage et l'enseignement.

- Il permet d'améliorer la mémoire de l'apprenant.

Étapes de l'apprentissage basé sur le cerveau.

Selon Jensen (2008) l'apprentissage basé sur le cerveau comprend sept étapes et chaque étape comprend des étapes définis:

1. Pré-exposition: Cette étape fournit au cerveau une vue d'ensemble du nouvel apprentissage avant de se creuser. La pré-exposition aide le cerveau à développer de meilleures cartes conceptuelles: Afficher un aperçu du nouveau sujet sur le babillard, apprendre à apprendre des stratégies de mémoire, Créer un environnement d'apprentissage par immersion solide et effectuer les ajustements nécessaires à mesure que l'enseignant poursuit les leçons.

2. Préparation: C'est l'étape à laquelle l'enseignant crée la curiosité ou l'excitation. Il est semblable à l'«ensemble d'anticipation», mais va plus loin dans la préparation de l'apprenant. Et créer une expérience «vous êtes là», donnant aux apprenants une véritable mise à la terre. Les encourager à exprimer comment ils estiment qu'il est ou n'est pas pertinent. Le cerveau apprend particulièrement bien des expériences concrètes d'abord.

3. Initiation et acquisition: Cette étape révèle la corrélation des sujets et soutien d'approfondir la compréhension chez les apprenants et les besoins de les intégrer dans des activités en classe pour une compréhension plus profonde et le feed-back avec les stratégies explicites et implicites, ce moyen est important de l'apprentissage. 
Efficacité de l'apprentissage basé sur le cerveau pour acquérir quelques règles grammaticales en français chez les étudiants du cycle secondaire

Prof.Dr. Sabri Eid Gad El Said \& Dr. El Adl Ahmed El Mahdi \& Racha Fouad Mohamed Abdel Hamid

4. Elaboration: Il s'agit de l'étape de traitement, il faut une véritable réflexion de la part des apprenants. C'est le moment de faire un sens intellectuel de l'apprentissage.

5. Encodage de la mémoire: Cette étape met l'accent sur l'importance du temps d'arrêt et du temps d'examen. Le cerveau apprend le plus efficacement au fil du temps, pas tout à la fois.

6. Vérification et la confiance en soi: Cette étape n'est pas seulement pour le bénéfice de l'enseignant, les apprenants ont besoin de confirmer euxmêmes leur apprentissage. L'apprentissage est mieux connu quand les étudiants possèdent un mode ou une métaphore concernant les nouveaux concepts ou matériaux.

7. Intégration: À ce stade, il est essentiel d'engager les émotions. Faites-en amusant, léger et joyeux. Cette étape inculque l'amour tout-important de l'apprentissage et l'évaluation des étapes précédentes.

\section{RÔLE DE L'ENSEIGNAN'T}

Selon Jensen (2008) l'enseignant joue un rôle essentiel dans l'apprentissage basé sur le cerveau:

- L'enseignant découvre les styles d'apprentissage et les méthodes de chaque apprenant et identifie la capacité du cerveau de l'apprenant.

- Il prépare le travail coopératif afin d'acquérir les méthodes d'interaction sociale et de fournir un environnement riche sans de la menace.

- Il donne l'occasion aux élèves d'analyser et d'installer des choses pour acquérir les compétences, à travers la discussion et le travail individuel et coopératif.

- Il compte tenu de l'occasion aux apprenants de la vigilance mentale et le brain-storming afin d'utiliser leurs cerveaux dans la découverte de l'environnement extérieur et de les inciter à l'apprentissage désiré.

- Il aide à activer les apprenants à l'intérieur et à l'extérieur de l'environnement d'apprentissage et leur permettent de reconnaître les dimensions spatiales des choses.

\section{RÔLE DE L'APPRENAN'T}

Kathlene et Specht (2003) déterminent le rôle de l'apprenant comme suit:

- L'apprenant peut être capable de participer avec les autres dans la prise de décision et de diriger lui-même les capacités du cerveau.

- Il peut traiter avec les méthodes de résolution des problèmes avec leurs différents types qui développent ses capacités intellectuelles.

- Il est conscient des processus de l'évaluation nécessaires d'apprendre individuellement et collectivement. 
Efficacité de l'apprentissage basé sur le cerveau pour acquérir quelques règles grammaticales en français chez les étudiants du cycle secondaire

Prof.Dr. Sabri Eid Gad El Said \& Dr. El Adl Ahmed El Mahdi \& Racha Fouad Mohamed Abdel Hamid

- Il participe à la réaction avec les établissements d'enseignement étrangers afin de pouvoir développer son cerveau.

- Il peut être capable d'utiliser tous les aspects physiques aux services le développement mental et cognitif du cerveau.

B- Les règles grammaticales

\section{IMPOR'TANCE DE IA GRAMMAIRE}

L'enseignement de la grammaire est très important dans l'apprentissage des langues étrangères parce qu'elle permet aux apprenants de communiquer, d'exprimer des situations orales ou écrites. D'autre parte, la grammaire a pour but de connaître l'emploi de la langue, de développer la production orale et écrite, et de permettre à l'apprenant d'éviter beaucoup d'erreurs.

Dans ce contexte, Cuq (2004) souligne que l'utilisation de la grammaire est considérée comme un moyen de faciliter l'apprentissage, non comme un objectif d'apprentissage, mais il faut augmenter chez l'apprenant l'idée qu'il lui est nécessaire de développer les règles grammaticales. De plus, l'enseignement de la grammaire peut donner d'une part, acquérir mieux de la langue à enseigner..

D'autre part, Charmeux (2006) montre que la grammaire permet à chacun de comprendre comment fonctionne la langue parlé et écrite, afin de donner la capacité sur la communication.

Parties de la grammaire

La grammaire se compose de deux parties fondamentales:

1. La syntaxe: Selon Gressive (2008) la syntaxe étudie les relations entre les mots dans la phrase: l'ordre des mots, l'accord sont des phénomènes de syntaxe. En outre, la syntaxe se distingue de la morphologie, étude de la formation interne des mots qui se fait à partir des plus petites unités appelées morphèmes.

2. La morphologie: Selon La Rousse (2002) la morphologie est la partie de la grammaire qui étudie la forme des mots et les variations de leurs désinences. Elle intéresse à étudier la forme de neuf espèces de mots: quatre espèces variables comme (l'article, le nom, le pronom et le verbe), quatre espèces invariables comme (l'adverbe, la préposition, la conjonction et l'interjection).

3. Types de la grammaire

1. La grammaire traditionnelle: La grammaire traditionnelle pour Moeschler et Auchlin (2000) est composée et comprend deux unités fondamentales sont le mot et la phrase. La phrase est un assemblage des mots qu'ont des sens complets, c'est-à-dire que la phrase constitue plusieurs propositions (phrase composée et phrase complexe). 
Efficacité de l'apprentissage basé sur le cerveau pour acquérir quelques règles grammaticales en français chez les étudiants du cycle secondaire

Prof.Dr. Sabri Eid Gad El Said \& Dr. El Adl Ahmed El Mahdi \& Racha Fouad Mohamed Abdel Hamid

2. La grammaire distributionnelle: Selon Canelas (2009) la grammaire distributionnelle construit une forme de description des unités linguistiques dans la phrase et elle s'agit de déterminer les éléments qui précédent et suivent l'unité. La méthode distributionnelle a une portée pratique intéressante qui porte sur les positions qui ont la place très importante dans les études de la langue.

3. La grammaire structurale: Selon Moeschler et Auchlin (2000) la grammaire structurale est organisée principalement en des deux idées principales:

- Les unités de rang: la première idée principale de la grammaire structurale est l'hiérarchie; les unités de la grammaire sont en relation hiérarchique.

- L'analyse en constituants immédiats: toute unité grammaticale complexe est analysable en unités de rang immédiatement inférieur.

4. La grammaire générative: Dans la grammaire générative, il y a plus de catégories:

- Catégories lexicales : verbe, nom, adjectif, adverbe.

- Catégories syntagmatiques : syntagme nominal, syntagme verbal, syntagme adjectival, syntagme préposition.

- Catégories non lexicales : préposition, pronom, complément, déterminant.

5. La grammaire transformationnelle: Fuchs et Le Goffic (2000) soulignent que la grammaire transformationnelle considère le phénomène d'ambiguïté est le fait qu'une phrase peut donner quelquefois plusieurs significatives différente.

6. La grammaire fonctionnelle: Clairis (1994) montre que La grammaire fonctionnelle est plutôt une méthode à l'opposé de la grammaire traditionnelle qui a des recettes et n'a pas de méthode. La grammaire comprend deux axes principaux: la morphologie et la syntaxe. Dans la grammaire fonctionnelle du français: La morphologie joue un rôle très important dans la présentation de la grammaire d'une langue comme le français.

7. La grammaire de texte: Charaudeau (1992) indique que le texte est la manifestation matérielle de la mise en scène d'un acte de communication, pour servir la communication de parole d'un locuteur donné. La grammaire de texte situe l'analyse au niveau du fonctionnement linguistique du texte, en mettant l'accent sur l'utilisation qui est faite des procédés linguistiques par rapport à la cohérence, à la cohésion du texte, aux liens qui existent entre les structures des phrases et le contexte dans lequel elles s'insèrent. 
Efficacité de l'apprentissage basé sur le cerveau pour acquérir quelques règles grammaticales en français chez les étudiants du cycle secondaire

Prof.Dr. Sabri Eid Gad El Said \& Dr. El Adl Ahmed El Mahdi \& Racha Fouad Mohamed Abdel Hamid

\section{'TECHNIOUES D'ENSEIGNEMEN'T DE GRAMMAIRE}

1. La grammaire explicite: Defays et Deltour (2003) montrent que la grammaire explicite est un ensemble de règles formelles que l'on a apprises de façon consciente et volontaire à l'école, dans un manuel, au moyen d'explications théoriques, d'exercices systématiques et d'efforts de mémorisation.

2. La grammaire implicite: Courtillon (2003) affirme que la grammaire implicite c'est que seule une connaissance implicite qui permet de la production. Elle est fondée sur l'élimination d'un méta-langage au profit de manipulations systématiques d'énoncés. Elle est basée sur une conception structurale de la langue.

3. La grammaire conceptualisation: Pour Besse et Porquier (1991) cette activité animant un esprit de réflexion, de déduction d'analyse, et de synthèse, afin d'objectif de faire de la classe un lieu d'échange et de communication, à travers les discussions qu'entretiennent les étudiants avec leur enseignant à propos des règles du fonctionnement de la structure rencontrée.

\section{ÉVALUATION DE IA GRAMMAIRE}

Cuq (1996) détermine plusieurs méthodes de l'évaluation de grammaire comme:

- Les exercices de compréhension: Ces exercices sont comme questionnaires à choix multiples. Ces exercices sont des manières d'évaluer la grammaire. Ils permettent de faire une évaluation simple et rapide. Ils peuvent appliquer ce que les élèves comprennent de grammaire.

- Les exercices structuraux: C'est le type le plus utilisé et connu d'exercices dans l'évaluation des règles grammaticales. Ces exercices structuraux peuvent classifier en exercices de répétition, de substitution, et de transformation.

- Les exercices d'expression: Ces exercices sont comme : matrice de textes, reformulation et réécriture de phrase ou du texte. Ils correspondent aux analyses grammaticales et logiques.

- Les exercices conceptualisation: Ces exercices impliquent la liberté d'exprimer c'est-à-dire, l'apprenant utilise les moyens qu'il peut comme: langue maternelle, langue étrangère, schémas et dessins d'exprimer de son apprentissage. Ces exercices font une grande place à l'activité métalinguistique.

- Les exercices sous forme de jeux linguistiques: Ils peuvent s'appliquer à toutes les unités grammaticales: ils regroupent les jeux grammaticaux, morphologiques ou syntaxiques, lexicaux, phonétiques et 
Efficacité de l'apprentissage basé sur le cerveau pour acquérir quelques règles grammaticales en français chez les étudiants du cycle secondaire

Prof.Dr. Sabri Eid Gad El Said \& Dr. El Adl Ahmed El Mahdi \& Racha Fouad Mohamed Abdel Hamid

orthographiques et permettent le maniement de certaines régularités de la langue ou la découverte de ses structures et caractéristiques ou la mémorisation de ses règles de fonctionnement.

\section{Étude expérimentale de la recherche}

I- Choix de l'échantillon de la recherche

L'échantillon de la recherche a compris 60 étudiants de la deuxième année secondaire aux écoles de Fakhr Al-Dakahlia officielle de langues à Mansourah au Gouvernorat d'Al- Dakahlia. La chercheuse a réparti l'échantillon en deux groupes: le groupe expérimental (30 étudiants) qui a appris selon l'apprentissage basé sur le cerveau et le groupe témoin (30 étudiants) qui a appris selon la méthode traditionnelle.

II- Outils de la recherche

1. Une grille des règles grammaticales: La chercheuse a analysé la méthode de français que les étudiants de la deuxième année secondaire aux écoles de langues étudient au deuxième semestre pour préparer une grille des règles grammaticales enseignées.

2. Test des règles grammaticales:

- Objectifs du test

- Évaluer quelques règles grammaticales chez les étudiants de la deuxième année secondaire aux écoles de langues.

- Mesurer l'efficacité de l'apprentissage basé sur le cerveau pour acquérir quelques règles grammaticales en français chez l'échantillon de la recherche.

- Description du test

Le test comprend cinq pages:

- La première page comprend les objectifs et les consignes du test.

- Les quatre autres pages sont consacrées à dix questions. Celles - ci mesurent les seize règles grammaticales:

- Étude pilote du test

L'étude pilote du test vise à vérifier la fidélité, la validité du test et la détermination de la durée du test. Pour réaliser ces objectifs, la chercheuse a appliqué un test sur un échantillon différent de celui de la recherche. L'échantillon est composé de 30 étudiants de la deuxième année secondaire aux écoles de langues.

- Fidélité du test

La fidélité du pré-post test fut mesurée à travers l'application par la formule de Pearson sur les étudiants (groupe de l'étude) dans le pré-test, sa valeur calculée fut $(0.83)$. Ce qui indique que le test est acceptable et peut être appliqué sur les étudiants (groupe de l'étude).

- Validité du test: 
Efficacité de l'apprentissage basé sur le cerveau pour acquérir quelques règles grammaticales en français chez les étudiants du cycle secondaire

Prof.Dr. Sabri Eid Gad El Said \& Dr. El Adl Ahmed El Mahdi \& Racha Fouad Mohamed Abdel Hamid

Pour calculer la validité du test, nous avons eu recours à deux manières:

- La chercheuse a présenté le test aux membres du jury composé de certains spécialistes en didactique du français et en langue français et certains orienteurs et enseignants du français. Ils ont décidé que le test est valide.

- La chercheuse a calculé la validité du test à partir de sa fidélité en appliquant la formule suivante:

la validité $=\sqrt{\text { la fidélité }}$

Cette validité est élevée. Donc, le test est valide.

la validité $=\longdiv { 0 . 8 3 = 0 . 9 1 }$

- Durée du test

Nous avons calculé la durée du test, selon la formule suivante:

$\mathbf{D}=$

Temps consommé par chaque élève pour tous les membres du groupe

Nombre du groupe

2700

$D=-=90$ minutes Alors, la durée du test est une heure et demie. 30

III- Préparation de deux unités expérimentales

1. Contenu de deux unités expérimentales

Chaque unité se compose de quatre leçons, trois leçons d'enseignement et une leçon de révision. Ces deux unités sont reformulées à la lumière de l'apprentissage basé sur le cerveau.

En ce qui concerne les activités de deux unités, la chercheuse a eu recours à quelques activités à la méthode de français et elle a aussi présenté d'autres activités qui aident à acquérir quelques règles grammaticales chez les étudiants de la deuxième année secondaire aux écoles de langues.

2. Enseignement de deux unités expérimentales

Après avoir appliqué le pré-test, l'enseignante a enseigné les deux unités expérimentales au groupe expérimental en suivant la démarche de l'apprentissage basé sur le cerveau. Elle a aussi enseigné les mêmes deux unités au groupe témoin selon la méthode traditionnelle.

\section{Résultats de la recherche}

I- Analyse statistique des résultats

- Pour vérifier la première hypothèse, on a calculé le Test $(T)$ pour déterminer les différences entre les moyennes des notes des étudiants de deux groupes au post- test en ce qui concerne les règles grammaticales. On peut déterminer ces résultats selon le tableau suivant: 
Efficacité de l'apprentissage basé sur le cerveau pour acquérir quelques règles grammaticales en français chez les étudiants du cycle secondaire

Prof.Dr. Sabri Eid Gad El Said \& Dr. El Adl Ahmed El Mahdi \& Racha Fouad Mohamed Abdel Hamid

\section{Tableau (1)}

Résultats du groupe témoin et du groupe expérimental au post- test

\begin{tabular}{|c|c|c|c|c|c|c|c|}
\hline Domaine & Groupe & $\mathbf{M}$ & $\mathbf{E}$ & D.L & T. & S. & $\eta^{2}$ \\
\hline \multirow[b]{2}{*}{ Articles } & Témoin & 2.8286 & .74698 & \multirow{10}{*}{68} & \multirow[b]{2}{*}{11.4} & \multirow{10}{*}{0.05} & \multirow[b]{2}{*}{0.66} \\
\hline & $\begin{array}{l}\text { Expérimenta } \\
\text { l }\end{array}$ & 4.9429 & .93755 & & & & \\
\hline \multirow[b]{2}{*}{ Prépositions } & Témoin & 1.9714 & .74698 & & \multirow[b]{2}{*}{11.2} & & \multirow[b]{2}{*}{0.65} \\
\hline & Expérimenta & 4.0000 & .90749 & & & & \\
\hline \multirow[b]{2}{*}{ Formes } & Témoin & 22.7143 & 2.29541 & & \multirow[b]{2}{*}{33.6} & & \multirow[b]{2}{*}{0.94} \\
\hline & $\begin{array}{l}\text { Expérimenta } \\
\text { l }\end{array}$ & 41.4571 & 2.36821 & & & & \\
\hline \multirow{2}{*}{$\begin{array}{l}\text { Temps et } \\
\text { leurs } \\
\text { adverbes }\end{array}$} & Témoin & 14.8571 & $\mathbf{1 . 7 6 8 0 6}$ & & \multirow[b]{2}{*}{15.0} & & \multirow[b]{2}{*}{0.77} \\
\hline & $\begin{array}{l}\text { Expérimenta } \\
\text { l }\end{array}$ & 21.5714 & 1.97463 & & & & \\
\hline \multirow[b]{2}{*}{ Total } & Témoin & 42.3714 & 3.63873 & & \multirow[b]{2}{*}{29.6} & & \multirow[b]{2}{*}{0.93} \\
\hline & $\begin{array}{l}\text { Expérimenta } \\
\text { l }\end{array}$ & 71.9714 & 4.65571 & & & & \\
\hline
\end{tabular}

Ce tableau montre que les valeurs de (T) aux domaines sont entre (11.2-33.6) et à la note totale (29.6). Donc, il existe une différence significative entre les moyennes des notes des élèves du groupe expérimental et celles du groupe témoin au post- test des règles grammaticales en faveur des élèves du groupe expérimental. Cette valeur est significative au niveau $(0.05)$. Par suite, la première hypothèse a été réalisée.

- Pour vérifier la deuxième hypothèse, on a calculé le Test $(T)$ pour déterminer les différences entre les moyennes des notes des étudiants du groupe expérimental au pré /post test des règles grammaticales. On peut déterminer ces résultats selon le tableau suivant:

Tableau (2)

Résultats du groupe expérimental au pré/post- test

\begin{tabular}{|c|c|c|c|c|c|c|c|}
\hline Domaine & Groupe & $\mathbf{M}$ & $\mathbf{E}$ & D.L. & T. & S. & $\eta 2$ \\
\hline \multirow{2}{*}{ Articles } & Pré-test & 2.9714 & .85700 & \multirow[b]{6}{*}{34} & \multirow[t]{2}{*}{9.9} & & \multirow[t]{2}{*}{0.74} \\
\hline & Post-test & 4.9429 & .93755 & & & & \\
\hline \multirow{2}{*}{ Prépositions } & Pré-test & 2.1429 & $\mathbf{. 8 4 5 1 5}$ & & \multirow[t]{2}{*}{8.7} & & \multirow[t]{2}{*}{0.69} \\
\hline & Post-test & 4.0000 & .90749 & & & & \\
\hline \multirow{2}{*}{ Formes } & Pré-test & 22.2857 & 2.72862 & & \multirow[t]{2}{*}{34.3} & & \multirow[t]{2}{*}{0.97} \\
\hline & Post-test & 41.4571 & 2.36821 & & & & \\
\hline
\end{tabular}


Efficacité de l'apprentissage basé sur le cerveau pour acquérir quelques règles grammaticales en français chez les étudiants du cycle secondaire

Prof.Dr. Sabri Eid Gad El Said \& Dr. El Adl Ahmed El Mahdi \& Racha Fouad Mohamed Abdel Hamid

\begin{tabular}{|c|c|c|c|c|c|c|c|}
\hline Domaine & Groupe & $\mathbf{M}$ & $\mathbf{E}$ & D.L. & T. & S. & $\eta 2$ \\
\hline \multirow{2}{*}{$\begin{array}{ll}\text { Temps } & \text { et } \\
\text { leurs } & \\
\text { adverbes } & \end{array}$} & Pré-test & 15.1429 & 2.15765 & & \multirow[b]{2}{*}{13.2} & \multirow{3}{*}{0.05} & \multirow[b]{2}{*}{0.84} \\
\hline & Post-test & 21.5714 & 1.97463 & & & & \\
\hline \multirow{2}{*}{ Total } & Pré-test & 42.5429 & 5.03717 & & \multirow{2}{*}{26.99} & & \multirow{2}{*}{0.96} \\
\hline & Post-test & 71.9714 & 4.65571 & & & & \\
\hline
\end{tabular}

Ce tableau montre que les valeurs de (T) aux domaines sont entre (8.7 - 34.3) et à la note totale (26.99). Donc, il existe une différence significative entre les moyennes des notes des étudiants du groupe expérimental au prépost test des règles grammaticales en faveur du post - test. Cette valeur est significative au niveau (0.05). Par suite, la deuxième hypothèse a été réalisée.

Pour vérifier la troisième hypothèse, on a calculé la proportion du gain modifié de Black et la proportion d'efficacité de McGogian. On peut déterminer ces résultats selon le tableau suivant:

Tableau(3)

Proportion du gain modifié de Black et proportion d'efficacité de McGogian.

\begin{tabular}{|l||l|l|l|l|}
\hline N.T. & M.N.Pré & M.N.Post. & P.G.M. & P.E. \\
\hline 90 & 42.5 & 71.97 & 1.2 & 62.0 \\
\hline
\end{tabular}

Ce tableau montre que la proportion du gain modifié de Black est (1.2) et la proportion d'efficacité de McGogian est (62.0). Ces valeurs indiquent qu'il existe une efficacité de l'apprentissage basé sur le cerveau pour l'acquisition des règles grammaticales chez les étudiants de la deuxième année secondaire aux écoles de langues. Par suite, la troisième hypothèse a été réalisée.

II- Interprétation des résultats

D'après les résultats déjà présentés, on peut conclure que l'apprentissage basé sur le cerveau est efficace pour acquérir les règles grammaticales chez l'échantillon de la recherche. Ce résultat peut être interprété selon les facteurs suivants:

- L'apprentissage basé sur le cerveau a aidé à développer des attitudes positives envers la grammaire ce qui a rendu les élèves plus actifs.

- Il a permis à développer la communication entre les élèves et l'enseignant qui a facilité la compréhension des règles grammaticales.

- Il a fourni l'atmosphère qui a favorisé l'amélioration de la capacité d'apprentissage. 
Efficacité de l'apprentissage basé sur le cerveau pour acquérir quelques règles grammaticales en français chez les étudiants du cycle secondaire

Prof.Dr. Sabri Eid Gad El Said \& Dr. El Adl Ahmed El Mahdi \& Racha Fouad Mohamed Abdel Hamid

- Il a encouragé efficacement les élèves à fonctionner leur cerveau lors de l'apprentissage.

- Il a aidé à améliorer la mémoire des élèves par la réflexion continue.

- Il a incité les élèves à prendre les décisions et à employer leurs capacités d'apprentissage.

- Il a accru la confiance de soi chez les élèves et les a incité à compter sur eux même pour accomplir les activités des règles grammaticales.

- Il a aidé les élèves à participer les idées et les opinions pour échanger les vues avec les autres.

- Les étapes de l'apprentissage basé sur le cerveau ont conduit les élèves à mieux penser et à réfléchir.

\section{III- Recommandations de la recherche}

D'après les résultats de cette recherche, on peut proposer les recommandations suivantes :

- S'intéresser à l'emploi de la stratégie de l'apprentissage basé sur le cerveau dans l'enseignement des règles grammaticales aux différents cycles éducatifs.

- Présenter des activités variées pour développer les règles grammaticales chez les apprenants.

- Avoir recours a des nouvelles méthodes dans l'enseignement du FLE qui favorisent l'interaction entre les apprenants et leurs enseignants.

- Fournir aux apprenants un feedback pendant l'enseignement des règles grammaticales pour corriger et éviter leurs erreurs.

- Évaluer les apprenants de façon continuelle qui les aident à accomplir leur apprentissage.

- Employer la stratégie de l'apprentissage basé sur le cerveau dans l'enseignement d'autres compétences langagières en classe du FLE.

IV-Suggestions de la recherche

Selon les résultats de cette recherche, on peut suggérer d'autres sujets de recherche :

- Utiliser l'apprentissage basé sur le cerveau à l'enseignement des compétences de l'expression écrite chez les élèves du cycle secondaire.

- Faire des comparaisons entre l'apprentissage basé sur le cerveau et le brainstorming pour mesurer leur efficacité en vue d'acquérir acquérir les règles grammaticales chez les apprenants aux différents cycles éducatifs.

- Élaborer un programme basé sur l'apprentissage basé sur le cerveau pour développer les compétences de la compréhension en lecture chez les étudiants du cycle universitaire. 
Efficacité de l'apprentissage basé sur le cerveau pour acquérir quelques règles grammaticales en français chez les étudiants du cycle secondaire

Prof.Dr. Sabri Eid Gad El Said \& Dr. El Adl Ahmed El Mahdi \& Racha Fouad Mohamed Abdel Hamid

- Elaborer unité proposée basée sur l'apprentissage hybride pour développer la compréhension orale chez les élèves au cycle préparatoire.

- Élaborer une unité proposée basée sur l'apprentissage par découverte pour acquérir les règles grammaticales chez les élèves du cycle primaire.

\section{Problématique de l'étude:}

La problématique de l'étude en cours réside dans la faiblesse des compétences en expression écrite créative en français chez les étudiants du cycle secondaire aux écoles de langues.

La problématique de l'étude se résume dans la question suivante:

"Quel est l'effet d'employer la stratégie du brainstorming sur le développement des compétences en expression écrite créative chez les étudiants des écoles secondaires de langues?".

A fin de répondre à cette question majeure, on la divise, en sousquestions suivantes:

1. Quelles sont les compétences de l'expression écrite créative, en français, nécessaires pour les étudiants des écoles secondaires de langues?

2. À quel degré les étudiants de $1^{\text {ère }}$ année secondaire des écoles de langues maîtrisent-ils ces compétences?

3. Quel est l'effet d'employer la stratégie du brainstorming sur le développement des compétences en expression écrite créative, en français, chez les étudiants des écoles secondaires de langues?

3. Hypothèses de l'étude:

La présente étude essaye de mettre en preuve les hypothèses suivantes:

1. Il y a des différences significatives statistiquement au niveau $(, 05 \geq)$ entre la moyenne des notes des étudiants du groupe expérimental et celles du groupe témoin au post-test en faveur du groupe expérimental au post-test.

2. Il y a des différences significatives statistiquement au niveau ( , $05 \geq$ ) entre la moyenne des notes des étudiants du groupe expérimental au pré/post-test en faveur du post-test.

4. Objectif de l'étude:

Cette étude vise à étudier le développement de quelques compétences de l'expression écrite créative en français à savoir la fluidité, la flexibilité, l'originalité et la sensibilité aux problèmes à travers l'emploi de la stratégie du brainstorming chez les étudiant de la $1^{\text {ère }}$ année du cycle secondaire à l'école expérimentale de langues à Mansourah.

4. Importance de l'étude:

L'importance de cette étude peut se résumer en:

1. aide les enseignants à bien accomplir leur tâche et surtout dans l'enseignement de l'expression écrite créative.

2. améliorer les capacités des étudiants dans l'expression écrite créative. 
Efficacité de l'apprentissage basé sur le cerveau pour acquérir quelques règles grammaticales en français chez les étudiants du cycle secondaire

Prof.Dr. Sabri Eid Gad El Said \& Dr. El Adl Ahmed El Mahdi \& Racha Fouad Mohamed Abdel Hamid

3. Offrir une unité dont le professeur peut utiliser dans les cours de l'expression écrite créative.

4. acquérir d'autres compétences langagières grâce à la technique du brainstorming.

5. permettre d'ouvrir à nos successeurs des horizons nouveaux de recherches portant sur les compétences langagières du français, en général, et celles de l'expression écrite créative en particulier.

6. promouvoir l'acquisition d'autres compétences langagières chez les étudiants grâce à la technique du brainstorming.

5. Limites de l'étude:

L'étude en cours se limite à:

1. un échantillon des étudiants de la $\mathbf{1}^{\text {ère }}$ année secondaire à l'école expérimentale de langues à Mansourah.

2. développer certaines compétences en expression écrite créative en employant la stratégie du brainstorming.

3. une unité d'enseignement proposée.

6. Echantillon de l'étude:

Notre échantillon se compose de trente étudiants parmi ceux de la $1^{\text {ère }}$ année secondaire des écoles expérimentales de langues à Mansourah. Ils ont étudié le français comme langue étrangère pendant trois ans successifs au cycle préparatoire. Cet échantillon se divise en deux groupes: l'un est expérimental, l'autre est témoin. Chaque groupe se compose de quinze étudiants. Le groupe témoin a étudié selon la méthode traditionnelle et le groupe expérimental a étudié selon la stratégie du brainstorming.

7. Outils de l'étude:

1. Grille de compétences d'expression écrite créative nécessaires aux étudiants de la $1^{\text {ère }}$ année secondaire.

2. Test de mesure des compétences de l'expression écrite créative des étudiants de la $1^{\text {ère }}$ année secondaire.

3. Certains thèmes à aborder avec les étudiants dans les séances de brainstorming.

4. Une unité proposée basée sur l'emploi de la stratégie du brainstorming.

8. Etapes de l'étude:

Pour répondre aux questions de l'étude, nous avons suivi les étapes suivantes:

1. Passer en revue les études antérieures portant sur:

- les stratégies d'enseignement/apprentissage surtout celle du brainstorming.

- l'expression écrite, en général, et créative en particulier.

2. Déterminer les compétences en expression écrite créative nécessaires 
Efficacité de l'apprentissage basé sur le cerveau pour acquérir quelques règles grammaticales en français chez les étudiants du cycle secondaire

Prof.Dr. Sabri Eid Gad El Said \& Dr. El Adl Ahmed El Mahdi \& Racha Fouad Mohamed Abdel Hamid

aux étudiants de la $\mathbf{1}^{\text {ere }}$ année secondaire des écoles expérimentales de langues à partir:

- de l'étude des objectifs de l'enseignement du français langue étrangère au cycle secondaire.

- des études antérieures.

- des analyses théoriques.

3. Elaborer une grille de compétences d'expression écrite créative nécessaires aux étudiants de la $1^{\text {ère }}$ année secondaire des écoles expérimentales de langues.

4. Elaborer un questionnaire contenant les thèmes proposés à aborder avec les étudiants de la1 ${ }^{\text {ère }}$ année secondaire des écoles expérimentales de langues.

5. Elaborer l'unité proposée basée sur l'emploi de la stratégie du brainstorming et visant à développer l'expression écrite créative chez les étudiants de la $1^{\text {ère }}$ année secondaire des écoles expérimentales de langues.

6. Elaborer le test de mesure de niveau des étudiants de la $1^{\text {ère }}$ année secondaire des écoles expérimentales de langues en expression écrite créative.

7. Soumettre les outils de l'étude aux experts et spécialistes (jury) pour vérifier la validité apparente des outils.

8. Modifier les outils d'après les points de vue des experts.

9. Choisir un échantillon de trente étudiants et le diviser en deux groupes.

10. Appliquer le pré-test en vue de vérifier le niveau du groupe expérimentale.

11. Appliquer l'unité proposée sur les étudiants de l'échantillon.

12. Appliquer le post-test sur les deux groupes.

13. Analyser statistiquement les notes des étudiants.

14. Interpréter les résultats de l'étude.

15. Présenter les suggestions et les recommandations de l'étude.

9. Terminologies de l'étude:

La stratégie:

De Villers (1992) définit la stratégie comme: l'art de planifier et de coordonner un ensemble d'opérations en vue d'atteindre un objectif. La stratégie du brainstorming:

Le dictionnaire (Robert Quotidien) indique que Le brainstorming "est

"Le nombre idéal dans la séance du brainstorming est 15 participants. 
Efficacité de l'apprentissage basé sur le cerveau pour acquérir quelques règles grammaticales en français chez les étudiants du cycle secondaire

Prof.Dr. Sabri Eid Gad El Said \& Dr. El Adl Ahmed El Mahdi \& Racha Fouad Mohamed Abdel Hamid

un mot anglais se composant de deux mots, le premier (brain) et l'autre (storming) et le brainstorming est une séance dont chaque personne donne ses opinions" (Legrain, 1996: 224).

Selon All-Quany et Al-Gamal, (2003) le brainstorming, c'est une stratégie qui examine un problème ou un sujet dont les experts regroupent pour arriver à une ou des solutions au problème posé à travers les discussions. Le but du Brainstorming est de produire le plus d'idées possibles, sur un thème donné, dans un minimum de temps sans critiquer et sans juger.

D'après le chercheur, le brainstorming est une stratégie que l'on utilise dans les cours de l'expression écrite créative pour produire le plus grand nombre des mots, des phrases, des idées et des paragraphes sur un sujet donné, dans un minimum de temps sans critiquer.

La compétence:

Les dictionnaires définissent la compétence comme: "une connaissance ou une capacité reconnue dans un domaine particulier" mais Robert indique que Chomsky définit la compétence comme la connaissance implicite, innée, que tout individu possède de sa langue" (Robert, 2008: 17).

Pendant que Rowr (1981) donne une autre définition de la compétence: c'est la capacité sociale, physique ou intellectuelle dont on apprend à travers la pratique, la répétition et la pensée.

L'expression écrite creative:

Le dictionnaire de didactique des langues définit l'expression écrite comme: une opération qui consiste à produire un message écrit en utilisant les signes sonores d'une langue (Gallisson, $R$ et Coste, D., 1976, 208).

Selon (Madkor, 2002: 252) l'expression écrite créative "est une expression des expériences émotionnelles d'une manière suggestive et intéressante" et L'écriture créative en donne l'occasion aux étudiants d'exprimer leurs sentiments, leurs pensées et leurs fantasmes.

Selon l'étude actuelle, on peut dire que l'expression écrite créative, c'est la note des lycéens dans le test de l'expression écrite créative en français à savoir la fluidité, la flexibilité, l'originalité et la sensibilité aux problèmes.

Résultat de l'étude:

Cette étude comprend deux hypothèses pour vérifier l'efficacité de la stratégie du brainstorming sur le développement des compétences de l'expression écrite créative en français chez les étudiants de la $1^{\text {ère }}$ année secondaire des écoles de langues qui étudient le français comme deuxième langue étrangère:

1- Il y a des différences significatives statistiquement au niveau $(, 05 \geq)$ entre la moyenne des rangs des notes des étudiants du groupe expérimental et 
Efficacité de l'apprentissage basé sur le cerveau pour acquérir quelques règles grammaticales en français chez les étudiants du cycle secondaire

Prof.Dr. Sabri Eid Gad El Said \& Dr. El Adl Ahmed El Mahdi \& Racha Fouad Mohamed Abdel Hamid

celles du groupe témoin au post-test en faveur du groupe expérimental au post-test en général.

2-Il y a des différences significatives statistiquement au niveau $(, 05 \geq)$ entre la moyenne des rangs des notes des étudiants du groupe expérimental au pré/post-test en faveur du groupe expérimental au post-test en général. Première hypothèse

Pour vérifier la $1^{\text {ère }}$ hypothèse de l'étude qui stipule qu'il y a des différences significatives statistiquement au niveau $(, 05 \geq)$ entre la moyenne des rangs des notes des étudiants du groupe expérimental et celles du groupe témoin au post-test en faveur du groupe expérimental au post-test en général et en ce qui concerne:

- L'aspect de fluidité.

- L'aspect de flexibilité.

- L'aspect d'originalité.

- L'aspect de sensibilité aux problèmes.

Nous avons utilisé le test de (Mann-Whitney) pour les groupes indépendants. Pour ces calculs, nous avons utilisé le programme statistique SPSS. Le tableau suivant montre le résultat de ce test:

Tableau $\mathrm{N}^{0}$ (1) montre

La comparaison entre les moyennes des rangs du groupe expérimental et celles du groupe témoin au pos-test

\begin{tabular}{|c|c|c|c|c|c|c|}
\hline Compétences & Groupe & $\mathbf{N}$ & $\mathbf{M}$ & S.R & $\mathbf{U}^{*}$ & $\mathbf{S}$ \\
\hline \multirow{2}{*}{ Fluidité } & Témoin & 15 & 8.00 & 120.00 & \multirow{2}{*}{.00} & \multirow{2}{*}{.01} \\
\hline & Expérimental & 15 & 23.00 & 345.00 & & \\
\hline \multirow{2}{*}{ Flexibilité } & Témoin & 15 & 10.90 & 163.50 & \multirow{2}{*}{43.50} & \multirow{2}{*}{.01} \\
\hline & Expérimental & 15 & 20.10 & 301.50 & & \\
\hline \multirow{2}{*}{ Originalité } & Témoin & 15 & 8.50 & 127.50 & \multirow{2}{*}{7.50} & \multirow{2}{*}{.01} \\
\hline & Expérimental & 15 & 22.50 & 337.50 & & \\
\hline \multirow{2}{*}{$\begin{array}{l}\text { Sensibilité } \\
\text { aux } \\
\text { problèmes }\end{array}$} & Témoin & 15 & 8.13 & 122.00 & \multirow[b]{2}{*}{2.00} & \multirow[b]{2}{*}{.01} \\
\hline & Expérimental & 15 & 22.87 & 343.00 & & \\
\hline \multirow{2}{*}{ Total } & Témoin & 15 & 8.00 & 120.00 & \multirow{2}{*}{.00} & \multirow{2}{*}{.01} \\
\hline & Expérimental & 15 & 23.00 & 345.00 & & \\
\hline
\end{tabular}

Légende:

N = Nombre d'étudiants.

$M=$ Moyenne de rangs. 
Efficacité de l'apprentissage basé sur le cerveau pour acquérir quelques règles grammaticales en français chez les étudiants du cycle secondaire

Prof.Dr. Sabri Eid Gad El Said \& Dr. El Adl Ahmed El Mahdi \& Racha Fouad Mohamed Abdel Hamid

S.R =Somme des rangs.

$\mathrm{U}=$ Valeur de (u).

S = nSignificative.

D'après le tableau précédent, on remarque que la moyenne du post-test du groupe expérimental est 8,00 et la moyenne du post-test du groupe témoin est 23,00. La valeur de (u) est ,00. Cela indique qu'il y a des différences significatives au niveau 0,01 entre les moyennes des rangs des notes du groupe expérimental qui étudie en utilisant la stratégie du brainstorming et les moyennes des rangs des notes du groupe du témoin qui étudie en utilisant la méthode traditionnelle en faveur du groupe expérimental au post-test en général et en ce qui concerne:

- L'aspect de fluidité.

- L'aspect de flexibilité.

- L'aspect d'originalité.

- L'aspect de sensibilité aux problèmes.

Le tableau précédent indique aussi que :

1- La différence entre les moyennes des rangs des notes des étudiants du groupe témoins et celles du groupe expérimental au post-test en général est significative au niveau de 0,01 en faveur du groupe expérimental.

2- La différence entre les moyennes des rangs des notes des étudiants du groupe témoins et celles du groupe expérimental au post-test en ce qui concerne l'aspect de sensibilité aux problèmes est significative au niveau de 0,01 en faveur du groupe expérimental.

3- La différence entre les moyennes des rangs des notes des étudiants du groupe témoins et celles du groupe expérimental au post-test en ce qui concerne l'aspect d'originalité est significative au niveau de 0,01 en faveur du groupe expérimental.

4- La différence entre les moyennes des notes des étudiants du groupe témoins et celles du groupe expérimental au post-test en ce qui concerne l'aspect de flexibilité est significative au niveau de 0,01 en faveur du groupe expérimental.

5- La différence entre les moyennes des rangs des notes des étudiants du groupe témoins et celles du groupe expérimental au post-test en ce qui concerne $L$ 'aspect de fluidité est significative au niveau de 0,01 en faveur du groupe expérimental.

Figure $\mathbf{N}^{\mathbf{0}}$ (1) 
Efficacité de l'apprentissage basé sur le cerveau pour acquérir quelques règles grammaticales en français chez les étudiants du cycle secondaire

Prof.Dr. Sabri Eid Gad El Said \& Dr. El Adl Ahmed El Mahdi \& Racha Fouad Mohamed Abdel Hamid

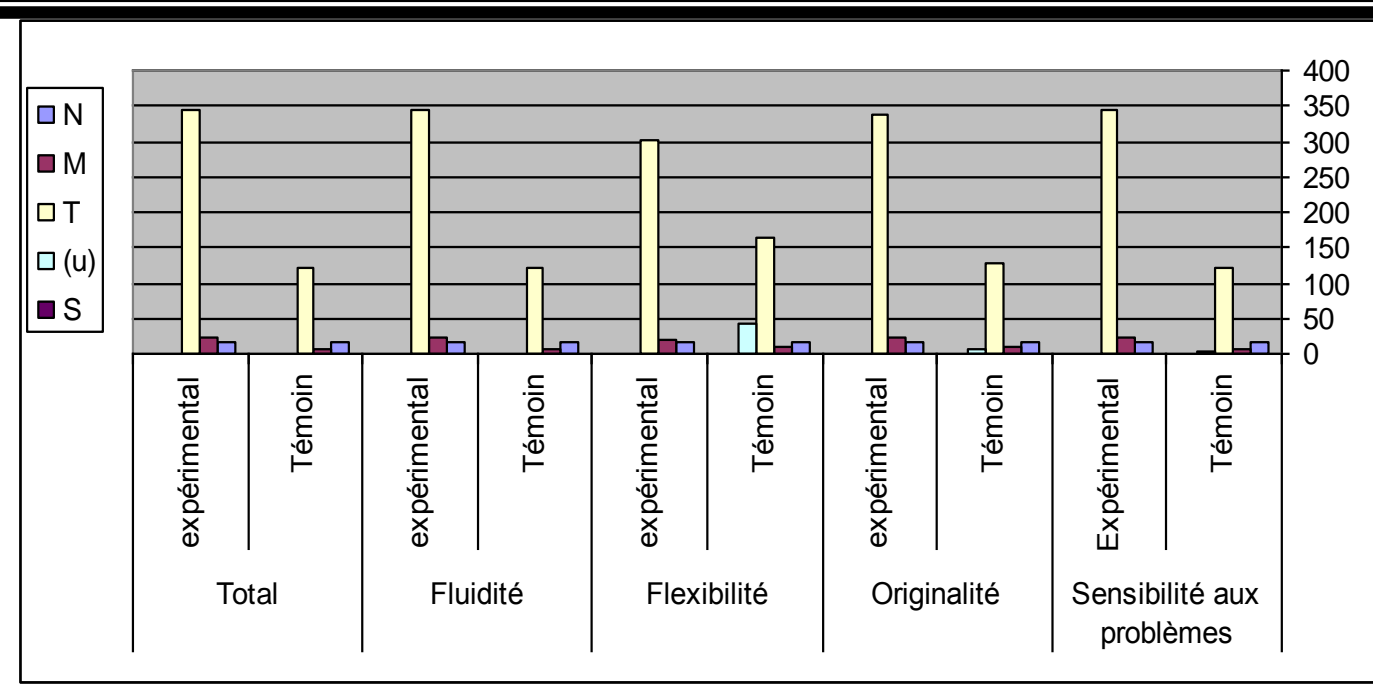

La figure $\mathrm{N}^{0}$ (1) résume le tableau précédent et montre la comparaison entre les moyennes des rangs du groupe expérimental et celles du groupe témoin au pos-test.

Donc, nous constatons la supériorité du groupe expérimental sur le groupe témoin en ce qui concerne l'aspect de sensibilité aux problèmes, l'aspect d'originalité, l'aspect de flexibilité et l'aspect de fluidité. Ce qui met en valeur l'efficacité du brainstorming et son influence sur le niveau du groupe expérimental en expression écrite créative. Par suite, la première hypothèse est justifiée:

Il y a des différences significatives statistiquement au niveau $(, 05 \geq)$ entre la moyenne des rangs des notes des étudiants du groupe expérimental et celles du groupe témoin au post-test en faveur du groupe expérimental au post-test en général et en ce qui concerne:

- L'aspect de fluidité.

- L'aspect de flexibilité.

- L'aspect d'originalité.

- L'aspect de sensibilité aux problèmes.

Donc, notre première hypothèse est confirmée, et on peut accepter la première hypothèse.

Deuxième hypothèse:

Pour vérifier la $2^{\text {ème }}$ hypothèse de l'étude qui stipule qu'il y a des différences significatives statistiquement au niveau $(, 05 \geq)$ entre la moyenne des rangs des notes des étudiants du groupe expérimental au pré/post-test en faveur du post-test en général et en ce qui concerne:

- L'aspect de fluidité.

- L'aspect de flexibilité.

- L'aspect d'originalité.

- L'aspect de sensibilité aux problèmes.

Nous avons utilisé le test de (Wilcoxon- signed Ranks test) pour le rang 
Efficacité de l'apprentissage basé sur le cerveau pour acquérir quelques règles grammaticales en français chez les étudiants du cycle secondaire

Prof.Dr. Sabri Eid Gad El Said \& Dr. El Adl Ahmed El Mahdi \& Racha Fouad Mohamed Abdel Hamid

moyen (groupes dépendants) non-paramétrique puisque le nombre des membres de l'échantillon est inférieur à 30 étudiants (15 étudiants) indépendants. Pour ces calculs, nous avons utilisé le programme statistique SPSS. Le tableau suivant montre le résultat de ce test:

Tableau $\mathrm{N}^{0}(2)$ montre

La comparaison entre les moyennes des rangs au pré/post-test d'expression écrite créative du groupe expérimental

\begin{tabular}{|c|c|c|c|c|c|c|}
\hline $\begin{array}{l}\text { Compéten } \\
\text { ces }\end{array}$ & $\mathbf{R}$ & $\mathbf{N}$ & $\mathbf{M}$ & S.R & $\mathbf{Z}^{*}$ & $\mathbf{S}$ \\
\hline \multirow{2}{*}{ Fluidité } & Négative (o-) & Zéro & Zéro & Zéro & \multirow{2}{*}{3.41} & \multirow{2}{*}{.01} \\
\hline & Positive (o+) & 15 & 8.00 & 120 & & \\
\hline \multirow{2}{*}{ Flexibilité } & Négative (o-) & 3 & 4 & 12 & \multirow{2}{*}{2.39} & \multirow{2}{*}{.01} \\
\hline & Positive $(\mathrm{o}+)$ & 10 & 7.90 & 79.00 & & \\
\hline \multirow{2}{*}{ Originalité } & Négative (o-) & Zéro & Zéro & Zéro & \multirow{2}{*}{3.47} & \multirow{2}{*}{,01 } \\
\hline & Positive (o+) & 15 & 8.00 & 120 & & \\
\hline \multirow{2}{*}{$\begin{array}{l}\text { Sensibilité } \\
\text { aux } \\
\text { problèmes }\end{array}$} & Négative (o-) & Zéro & Zéro & Zéro & \multirow[b]{2}{*}{3.23} & \multirow[b]{2}{*}{.01} \\
\hline & Positive (o+) & 13 & 7.00 & 19 & & \\
\hline \multirow{2}{*}{ Total } & Négative (o-) & Zéro & Zéro & Zéro & \multirow{2}{*}{3.41} & \multirow{2}{*}{.01} \\
\hline & Positive (o+) & 15 & 8.00 & 120 & & \\
\hline
\end{tabular}

Légende:

$\mathbf{R}=$ Les rangs.

$\mathrm{N}=$ Nombre d'étudiants.

$M=$ Moyenne des rangs.

S.R = Somme des rangs.

$Z=$ Valeur de $(Z)$.

$S=$ Significative.

En consultant le tableau $\mathbf{N}^{0}(12)$, nous pouvons remarquer que la moyenne des rangs du pré/post-test du groupe expérimental est entre zéro et 8,00. Le total des rangs est du pré/post-test du groupe expérimental est entre zéro et 120. La valeur de $(Z)$ est 3.41. Cela indique qu'il y a des différences significatives au niveau 0,01 entre les moyennes des rangs des notes du groupe expérimental qui étudie en utilisant la stratégie du brainstorming et les moyennes des rangs des notes du groupe du témoin qui étudie en utilisant la méthode traditionnelle en faveur du groupe expérimental au post-test en général et en ce qui concerne:

- L'aspect de fluidité.

- L'aspect de flexibilité.

- L'aspect d'originalité. 
Efficacité de l'apprentissage basé sur le cerveau pour acquérir quelques règles grammaticales en français chez les étudiants du cycle secondaire

Prof.Dr. Sabri Eid Gad El Said \& Dr. El Adl Ahmed El Mahdi \& Racha Fouad Mohamed Abdel Hamid

- L'aspect de sensibilité aux problèmes.

Le tableau ci-dessus montre qu':

1- Il y a une grande différence significative entre les moyennes des rangs des notes des étudiants du groupe expérimental au pré/post-test en faveur du post-test.

2- Il y a une grande différence significative entre les moyennes des rangs des notes des étudiants du groupe expérimental au pré/post-test en faveur du post-test en ce qui concerne l'aspect de sensibilité aux problèmes du test d'expression écrite créative.

3- Il y a une grande différence significative entre les moyennes des rangs des notes des étudiants du groupe expérimental au pré/post-test en faveur du post-test en ce qui concerne l'aspect d'originalité du test d'expression écrite créative.

4- Il y a une grande différence significative entre les moyennes des rangs des notes des étudiants du groupe expérimental au pré/post-test en faveur du post-test en ce qui concerne l'aspect de flexibilité du test d'expression écrite créative.

5- Il y a une grande différence significative entre les moyennes des rangs des notes des étudiants du groupe expérimental au pré/post-test en faveur du post-test en ce qui concerne l'aspect de fluidité du test d'expression écrite créative.

D'après les résultats précédents, on observe que l'utilisation de la stratégie du brainstorming avec le groupe expérimental a contribué positivement à développer certaines compétences en expression écrite créative chez eux.

Figure $\mathbf{N}^{0}(2)$

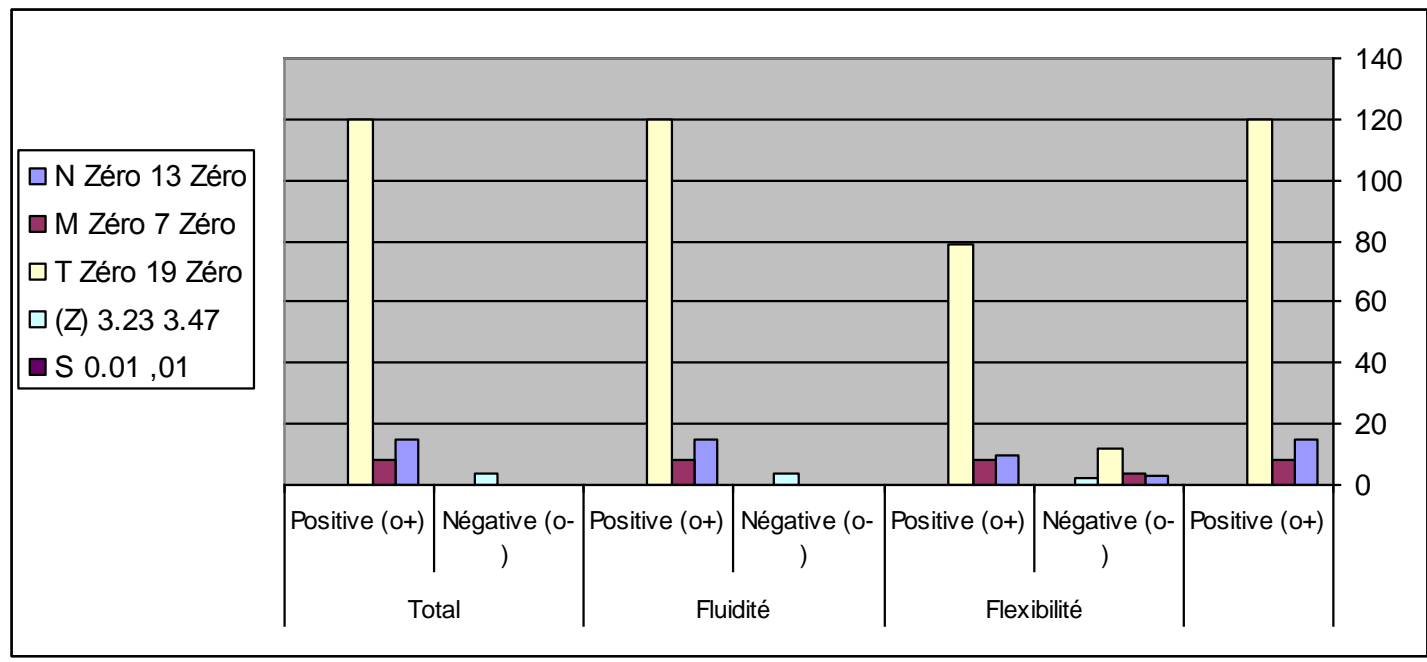

La figure $\mathbf{N}^{0}$ (2) résume le tableau précédent et montre la comparaison entre la comparaison entre les moyennes des rangs au pré/post-test d'expression écrite créative du groupe expérimental.

Par suite, la deuxième hypothèse est justifiée: 
Efficacité de l'apprentissage basé sur le cerveau pour acquérir quelques règles grammaticales en français chez les étudiants du cycle secondaire

Prof.Dr. Sabri Eid Gad El Said \& Dr. El Adl Ahmed El Mahdi \& Racha Fouad Mohamed Abdel Hamid

Il y a des différences significatives statistiquement au niveau $(, 05 \geq)$ entre la moyenne des rangs des notes des étudiants du groupe expérimental au pré/post-test en faveur du groupe expérimental au post-test en général et en ce qui concerne:

- L'aspect de fluidité.

- L'aspect de flexibilité.

- L'aspect d'originalité.

- L'aspect de sensibilité aux problèmes.

Donc, notre deuxième hypothèse est confirmée, et on peut accepter la deuxième hypothèse.

Après l'analyse statistique des résultats obtenus dans le test d'expression écrite créative, il est important de déterminer l'effet d'employer la stratégie brainstorming sur le développement des compétences en expression écrite créative chez le groupe expérimental.

2. Calcul de l'effet de l'unité proposé:

Pour vérifier l'effet d'employer la stratégie brainstorming sur le développement des compétences de l'expression écrite créative en français chez les membres de l'échantillon de l'étude, le chercheur a calculé le carré d'Eta $\left(\eta^{2}\right)$ qui indique le degré de l'effet sur le développement des compétences choisies, selon l'équation suivante:

$$
\boldsymbol{\eta}^{2}=\frac{\mathrm{T} 2}{\mathrm{~T} 2+\mathrm{D} \cdot \mathrm{L}}
$$

Le tableau suivant montre les résultats

Tableau $\mathrm{N}^{\mathbf{0}}(3)$ montre

Le degré de l'effet de l'emploi du brainstorming sur le développement des compétences de l'expression écrite créative

\begin{tabular}{|l|l|l|l||}
\hline Compétence & Valeur $(\mathbf{t})$ & $\eta 2$ & D.E \\
\hline Fluidité & 15.91 & .95 & Élévé \\
\hline Flexibilité & 2.94 & .38 & Elevé \\
\hline \hline Originalité & $\mathbf{1 0 . 2 2}$ & .88 & Elevé \\
\hline $\begin{array}{l}\text { Sensibilité aux } \\
\text { problèmes }\end{array}$ & $\mathbf{6 . 1 0}$ & .73 & Elevé \\
\hline Total & $\mathbf{2 0 . 7 9}$ & .97 & Élévé \\
\hline
\end{tabular}

Légende:

$\eta^{2}=$ Carré d'Eta.

D.E =Degré d'effet

Le tableau $\mathrm{N}^{0}(3)$ indique que les valeurs de $(\eta 2)$ sont entre $(, 38-, 95)$ en 
Efficacité de l'apprentissage basé sur le cerveau pour acquérir quelques règles grammaticales en français chez les étudiants du cycle secondaire

Prof.Dr. Sabri Eid Gad El Said \& Dr. El Adl Ahmed El Mahdi \& Racha Fouad Mohamed Abdel Hamid

ce qui concerne les compétences de sensibilité aux problèmes, les compétences d'originalité, les compétences de flexibilité et les compétences de fluidité tandis que la valeur de $(\eta 2)$ pour le total des compétences est (,97) et cela signifie que le degré d'effet au niveau $(1,00)$ est élevé ${ }^{(1)}$.

Ces valeurs indiquent qu'il y a un effet efficace du brainstorming sur le développement des compétences de l'expression écrite créative en français chez les étudiants de la première année secondaire des écoles de langues qui étudient le français comme deuxième langue étrangère.

Figure $\mathbf{N}^{\mathbf{0}}(\mathbf{3})$

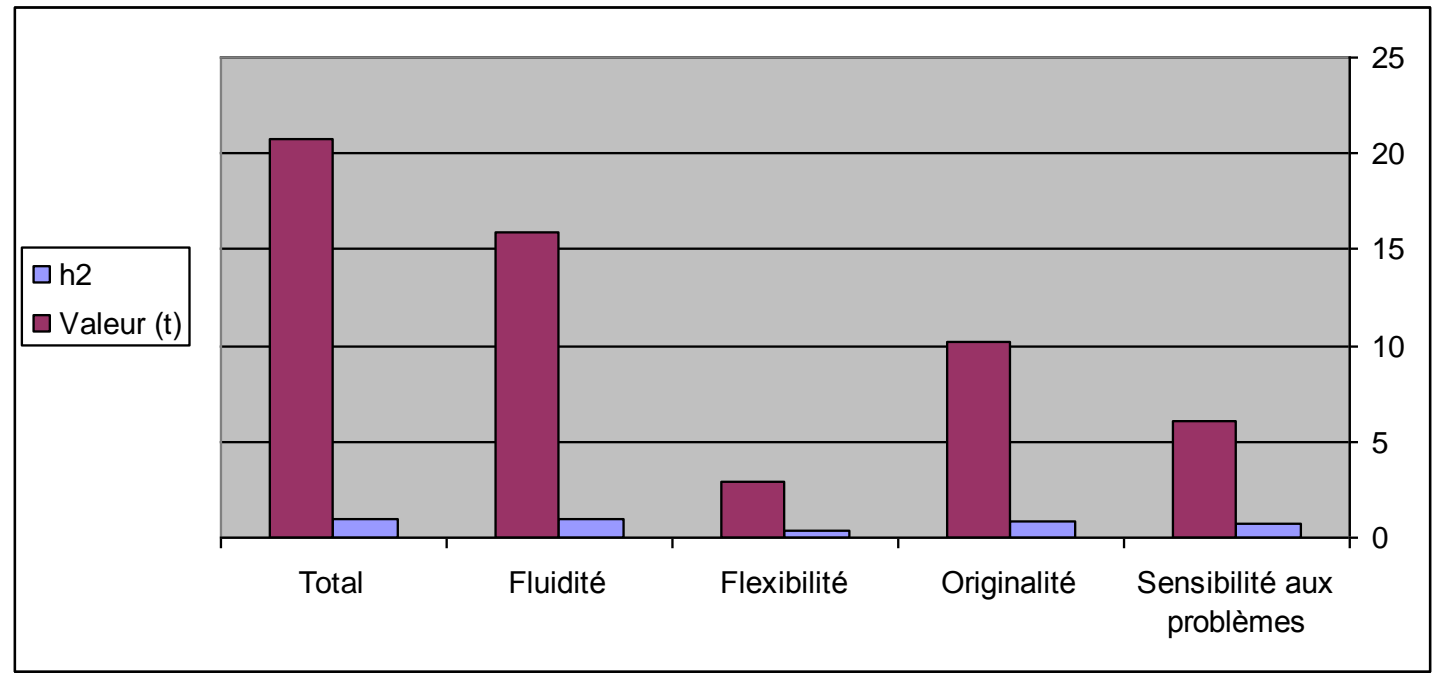

La figure $\mathrm{N}^{\mathbf{0}}(3)$ résume le tableau précédent et montre le degré de l'effet de l'emploi du brainstorming sur le développement des compétences de l'expression écrite créative.

\section{Commentaire général}

Après l'enseignement de l'unité proposé dans les séances du brainstorming pour le groupe expérimental et d'après les résultats déjà présentés, nous avons constater un progrès au niveau des étudiants du groupe expérimental en ce qui concerne les compétences de l'expression écrite créative en général, ensuite dans l'aspect de la sensibilité aux problèmes, l'aspect d'originalité, l'aspect de flexibilité et enfin dans l'aspect de fluidité et en même temps il n'y a aucun progrès au niveau des étudiants du groupe témoin en ce qui concerne les mêmes compétences.

Cela indique la réelle efficacité du brainstorming sur le développement des compétences de l'expression écrite créative en français chez l'échantillon de l'étude. 
Efficacité de l'apprentissage basé sur le cerveau pour acquérir quelques règles grammaticales en français chez les étudiants du cycle secondaire

Prof.Dr. Sabri Eid Gad El Said \& Dr. El Adl Ahmed El Mahdi \& Racha Fouad Mohamed Abdel Hamid

Cela nous amène à conclure que les résultats obtenus sont encourageants et que l'enseignement de l'unité, élaboré par le chercheur, en employant le brainstorming, a porté ces fruits.

Ces résultats s'accordent avec les résultats des études suivantes:

- L'étude de NIKI, P. (2000),

- L'étude de KHALIL, H. (2005),

- L'étude de ABD EL SALAM, M. (2007),

- L'étude de AL-ADL, W. (2010).

Toutes ces études ont confirmé l'efficacité du brainstorming sur le développement de toutes les compétences de la langue et surtout l'expression écrite créative.

Ce résultat peut être interprété comme suit:

La stratégie du brainstorming a beaucoup d'avantages, il se caractérise par ces caractères:

- La stratégie du brainstorming est facile d'application.

- Elle développe les compétences de la pensée chez les apprenants.

- Elle développe la confiance en soi.

- Elle développe la capacité d'exprimer les idées librement.

- Elle fait apparaître les idées créatives.

- Elle contribue à créer une atmosphère qui favorise l'acquisition de la langue dans un contexte plus naturel. Les étudiants sont donc plus enclins à apprendre et à fournir l'effort nécessaire.

- La stratégie du brainstorming aide à développer les attitudes positives envers la matière enseignée en rendant les étudiants plus motivés et maximise leur acquisition des compétences de l'expression écrite créative.

- Elle répond aux besoins des apprenants, et elle rend aussi l'enseignement plus efficace.

- Elle a le mérite d'être simple et accessible.

- Elle requiert peu de temps de la part des participants

- Elle permet de générer un grand nombre d'idées.

- L'accent est mis sur l'apprentissage des étudiants, plutôt que sur l'enseignement du professeur.

- La rétroaction aide les étudiants à se corriger pendant le travail et à se communiquer.

- L'interaction entre les étudiants au sein des groupes leur donne l'occasion d'échanger leurs connaissances et leurs expériences en créant un climat favorable pour développer les compétences de l'expression écrite créative.

En outre, Il y a plusieurs raisons derrière la popularité des remueméninges: 
Efficacité de l'apprentissage basé sur le cerveau pour acquérir quelques règles grammaticales en français chez les étudiants du cycle secondaire

Prof.Dr. Sabri Eid Gad El Said \& Dr. El Adl Ahmed El Mahdi \& Racha Fouad Mohamed Abdel Hamid

- Tout le monde se sent impliqué, avec la possibilité d'exprimer ouvertement son opinion.

- Il génère des résultats visibles grâce aux feuilles mobiles qui remplissent rapidement la pièce.

- Il se passe d'habitude dans une ambiance de bonne humeur et éloigné du lieu de travail.

- Il permet aux gens d'être créatifs et de penser au-delà des limites.

- Il encourage l'esprit d'équipe et crée un sentiment d'acceptation collégiale des données produites.

Cela indique l'effet positif de la stratégie du brainstorming sur le développement des compétences de l'expression écrite créative chez les étudiants de l'échantillon et révèle que l'enseignement du programme proposé basé sur la stratégie du brainstorming a donné ses fruits.

Donc, le chercheur a répondu à la question (4) suivante:

"Quel est l'effet de la stratégie du brainstorming sur le développement des compétences sur le développement des compétences en expression écrite créative chez les étudiants des écoles secondaires de langues?"

Conclusion

Dans ce chapitre, le chercheur a discuté la mise en pratique de cette étude expérimentale. Une question principale et deux hypothèse ont été émises; les résultats ont répondu d'une manière détaillée à la question principale de l'étude: "Quel est l'effet de la stratégie du brainstorming sur le développement des compétences sur le développement des compétences en expression écrite créative chez les étudiants des écoles secondaires de langues?

Pour vérifier la crédibilité et l'utilité des hypothèses de l'étude; le chercheur a appliqué le test d'expression écrite en français sur les deux groupes de l'étude après avoir expérimenté la stratégie du brainstorming sur le groupe expérimental. Le chercheur a calculé les moyennes des rangs des notes des étudiants; puis il a appliqué le test de (Wilcoxon- signed Ranks test) pour les moyens (groupes dépendants). Ensuite, il a calculé le carré d'Etta $\left(\eta^{2}\right)$ qui indique le degré de l'effet.

Les résultats ont affirmé la validité des hypothèses de l'étude, et on a trouvé des différences significatives statistiquement au niveau entre la moyenne des rangs des notes des étudiants du groupe expérimental et celles du groupe témoin au post-test en faveur du groupe expérimental au posttest en général et en ce qui concerne (l'aspect de sensibilité aux problèmes, l'aspect d'originalité, l'aspect de flexibilité, l'aspect de fluidité). (Hypothèse 1).

Il s'avère aussi de cette étude qu'il y a des différences significatives statistiquement entre la moyenne des rangs des notes des étudiants du 
Efficacité de l'apprentissage basé sur le cerveau pour acquérir quelques règles grammaticales en français chez les étudiants du cycle secondaire

Prof.Dr. Sabri Eid Gad El Said \& Dr. El Adl Ahmed El Mahdi \& Racha Fouad Mohamed Abdel Hamid

groupe expérimental au pré/post-test en faveur du groupe expérimental au post-test en général et en ce qui concerne (l'aspect de sensibilité aux problèmes, l'aspect d'originalité, l'aspect de flexibilité, l'aspect de fluidité). (Hypothèse 2).

Le chercheur peut donc conclure que ce programme a pu atteindre ses objectifs. Il peut aider les étudiants à développer leurs compétences de la l'expression écrite créative.

En effet, les résultats de l'étude ont mis l'accent sur l'efficacité de la stratégie du brainstorming sur le développement de certaines compétences en expression écrite créative en langue française.

Enfin, le chercheur propose un certain nombre de suggestions et de recommandations qui peuvent conduire au développement des compétences en expression écrite créative chez les étudiants du cycle secondaire.

Recommandations de l'étude de:

À la lueur des résultats de la présente étude, on pourrait recommander

- S'intéresser à l'entraînement des apprenants aux compétences de l'expression écrite créative dans les différents cycles éducatifs en général et au niveau secondaire en particulier.

- Consacrer un portfolio d'activités à chaque apprenant pour inscrire ses écriture variées en vue de développer sa capacité à écrire d'une manière créative.

- S'intéresser à l'entraînement des apprenants à la stratégie du brainstorming et aux compétences de l'expression écrite créative dans les situations d'apprentissage.

- Utiliser la stratégie du brainstorming à traiter des thèmes de la vie quotidienne en vue de motiver les apprenants à donner des idées variées sans craindre de faire des erreurs.

- Consacrer un cours par semaine à une séance de brainstorming durant laquelle les apprenants seraient invités à proposer un maximum d'idées sur un thème ou un problème donné pour les habituer à utiliser cette stratégie.

- S'intéresser aux activités langagières et aux différentes stratégies efficaces qui facilitent l'enseignement/apprentissage du français et répondent aux besoins de différents niveaux d'étudiants.

- Développer les programmes de formation (initiale et continue) des futurs-enseignants à la lueur des tendances modernes de l'enseignement.

- Entraîner les apprenants à utiliser et à bien choisir la stratégie adéquate à la situation d'apprentissage.

- La nécessité de donner la priorité à l'écrit et particulièrement à 
Efficacité de l'apprentissage basé sur le cerveau pour acquérir quelques règles grammaticales en français chez les étudiants du cycle secondaire

Prof.Dr. Sabri Eid Gad El Said \& Dr. El Adl Ahmed El Mahdi \& Racha Fouad Mohamed Abdel Hamid

l'expression écrite créative.

- La nécessité d'utiliser les nouvelles stratégies pour enseigner l'expression écrite créative aux écoles. On doit examiner ces stratégies pour voir si elles sont adéquates aux étudiants.

- Pour bien acquérir les compétences de l'expression écrite créative, les étudiants doivent pratiquer chaque compétence et effectuer plusieurs exercices sur chaque compétence sous la supervision de l'enseignant.

Recherches proposées:

Nous pouvons également, à travers les résultats obtenus, proposer certaines études suivantes en didactique de la langue française:

1. Une étude pour diagnostiquer les problèmes principaux qui affrontent les étudiants du cycle secondaire en expression écrite créative et leur remède.

2. Une étude comparative entre le brainstorming et d'autres techniques sur le développement de l'expression écrite créative chez les étudiants du cycle secondaire.

3. Une étude pour connaître l'effet de l'utilisation du brainstorming sur le rendement des étudiants et sur l'amélioration de leurs attitudes vers la langue française.

4. Utiliser la stratégie du brainstorming pour développer les compétences de la pensée critique et de la pensée créative en français.

5. Vérifier l'effet de la stratégie du brainstorming sur l'acquisition des étudiants et sur le développement de leurs attitudes envers la langue française.

6. Présenter des études basées sur les activités et la stratégie du brainstorming pour développer les différentes compétences de la langue française chez les étudiants de différents cycles éducatifs.

7. Effectuer un nombre d'études pour développer les compétences de l'expression écrite créative dans tous les cycles éducatifs et les niveaux langagiers. 
Efficacité de l'apprentissage basé sur le cerveau pour acquérir quelques règles grammaticales en français chez les étudiants du cycle secondaire

Prof.Dr. Sabri Eid Gad El Said \& Dr. El Adl Ahmed El Mahdi \& Racha Fouad Mohamed Abdel Hamid

\section{Bibliographie}

1. ABD EL-GHANI, Gh. (2004): Efficacité d'utilisation des activités langagières communicatives pour le développement de certaines compétences de l'expression écrite en français chez les étudiants du cycle préparatoires, Thèse de Magistère, Faculté de Pédagogie, Université de Mansourah.

2. ABD EL-GHANY, Ch. (2003): L'efficacité d'une stratégie proposée basée sur le style de l'exercice guide et la résolution des problèmes dans le développement de quelques compétences de l'expression écrite libre chez les étudiants de $4^{\text {ème }}$ année de section de français à la faculté de pédagogie de Tanta, Thèse de Magistère, Faculté de Pédagogie, Université de Tanta.

3. ALI, M. (1994): Les fautes communes en expression chez étudiants du département de français, à la faculté de pédagogie, Thèse de Magistère. Faculté de pédagogie, Université de Zagazig.

4. CANAC, A. (2001): La créativité dans l'enseignement, Rhône: CEFEDEM RHONE-ALPESONE.

5. CHENDI, W. (2010): L'étude de l'activité théâtrale sur le développement des compétences de l'écriture créative chez les apprenants du français langue seconde, Mémoire de Magistère, Faculté des Sciences de l'éducation au Canada, Université de Moncton, p. 2.

6. CHENDI, W. (2010): L'étude de l'activité théâtrale sur le développement des compétences de l'écriture créative chez les apprenants du français langue seconde, Mémoire de Magistère, Faculté des Sciences de l'éducation, Université de Moncton, p. 2.

7. DE VILLERS, M-E., (1992): Multi dictionnaire des difficultés de la langue française, 2 édition, Québec.

8. ENANY, H. (2012): Efficacité de l'utilisation du cycle d'apprentissage dans le développement de certaines compétences de l'écriture en français comme première langue étrangère chez les étudiants de la première année secondaire dans les instituts Azhariens, Thèse de Magistère, Faculté de Pédagogie, Universitéde Zagazig.

9. ENANY, H. (2012): Efficacité de l'utilisation du cycle d'apprentissage dans le développement de certaines compétences de l'écriture en français comme première langue étrangère chez les étudiants de la première année secondaire dans les instituts Azhariens, Thèse de Magistère, Faculté de Pédagogie, Université de Zagazig.

10.IBRAHIM, H. (1995): Programme proposé en vue de développer des compétences de la performance écrite en français $2^{\text {ème }}$ langue étrangère chez les élèves du cycle secondaire général, Thèse de Magistère, Faculté de Pédagogie, Université de Zagazig. 
Efficacité de l'apprentissage basé sur le cerveau pour acquérir quelques règles grammaticales en français chez les étudiants du cycle secondaire

Prof.Dr. Sabri Eid Gad El Said \& Dr. El Adl Ahmed El Mahdi \& Racha Fouad Mohamed Abdel Hamid

11.IBRAHIM, H. (1998): Effet d'un programme proposé à la lueur de l'approche des textes littéraires sur le développement des compétences de l'expression créative et de la réflexion critique chez les étudiants du département du français à la faculté de Pédagogie, Thèse de Doctorat, Faculté de Pédagogie, Université de Zagazig.

12.JARVIS, P. (1999): International dictionary of adult and continuing education, London: Kogan.

13.KHALIL, H. (2005): Effet d'employer «Le brainstorming» sur le développement des compétences de l'expression orale en français chez les étudiants des écoles secondaire de langues, Thèse de Magistère, Faculté de Pédagogie, Université de Zagazig.

14.LEGRAIN, M. (1996): Dictionnaire le robert quotidien, Paris.

15.MARTINET, A. (1996): Éléments de linguistique générale, $4^{\text {ème }}$ édition, Paris: Armand Colin.

16.MOGAHED, M. (2006): The effectiveness of using the process writing approach in developing The E. F. L. writing Skill in AL-Azhar secondary stage students and their attitudes toward it, Thesis of Master, Faculty of education - Mansourah University.

17.MOGAHED, M. (2006): The effectiveness of using the process writing approach in developing The E. F. L. writing Skill in AL-Azhar secondary stage students and their attitudes toward it, Thesis of Master, Faculty of education, Mansourah University.

18.NAWAR, H. (2004): L'efficacité de l'apprentissage coopératif et de l'enseignement individualisé sur le développement de l'aspect grammatical en expression écrite chez les étudiants des facultés de pédagogie, Thèse de Magistère. Faculté de pédagogie -Université de Zagazig, p. 67.

19.NAWAR, H. (2004): L'efficacité de l'apprentissage coopératif et de l'enseignement individualisé sur le développement de l'aspect grammatical en expression écrite chez les étudiants des facultés de pédagogie, Thèse de Magistère. Faculté de pédagogie, Université de Zagazig.

20.NIKI, P. (juillet-août 2000): "Enseigner en chantant", Le français dans le monde, $N^{0}$. (311), p. 39.

21.OMAR, H. (2007): Efficacité de l'utilisation de l'approche globale de la langue sur le développement des compétences de la lecture et de l'expression écrite en français chez les étudiants de la première année secondaire azharienne, Thèse de Magistère, Faculté de Pédagogie, Université d'Al-Azhar.

22.OMAR, H. (2007): Efficacité de l'utilisation de l'approche globale de la langue sur le développement des compétences de la lecture et de l'expression écrite en français chez les étudiants de la première année 
Efficacité de l'apprentissage basé sur le cerveau pour acquérir quelques règles grammaticales en français chez les étudiants du cycle secondaire

Prof.Dr. Sabri Eid Gad El Said \& Dr. El Adl Ahmed El Mahdi \& Racha Fouad Mohamed Abdel Hamid

secondaire azharienne, Thèse de Magistère, Faculté de Pédagogie, Université d'Al-Azhar.

23.ROBERT, J.P. (2008): L'essentiel francais, dictionnaire pratique de didactique du FLE, $2^{\text {ème }}$ édition, Paris: ophrys.

24.ROWR, D. (1981): A dictionary of education, London: Harper \& Row Publishers.

25.Tim, K. (1996): Inhibition within idea generating groups: an alternative method of brainstorming.

26.YOUSSEF, A. (2011): Efficacité de l'utilisation de la stratégie de l'apprentissage coopératif pour développer les compétences créatives à la lecture et à l'écriture en français chez les étudiants du département de français, Thèse de Doctorat, Faculté de Pédagogie, Université d'Al-Azhar.

27.YOUSSEF, A. (2011): Efficacité de l'utilisation de la stratégie de l'apprentissage coopératif pour développer les compétences créatives à la lecture et à l'écriture en français chez les étudiants du département de français, Thèse de Doctorat, Faculté de Pédagogie, Université d'Al-Azhar.

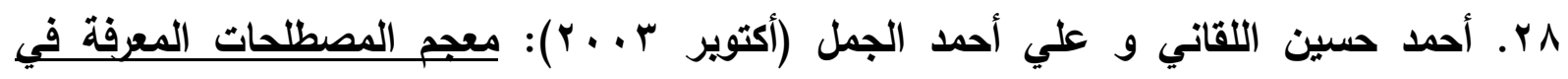

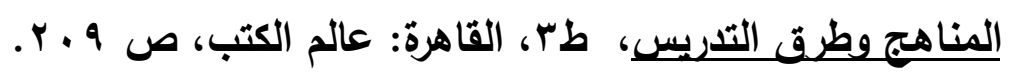

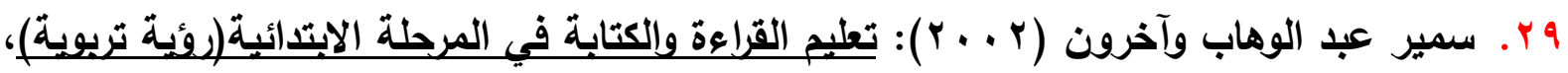

المنصورة: الدقهلية للطباعة وإلنشر.

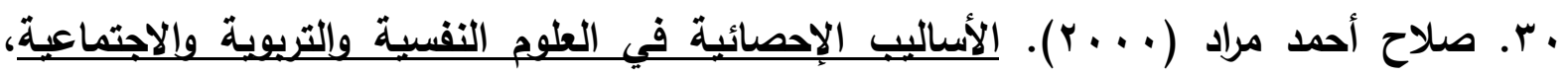

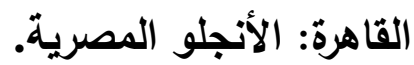

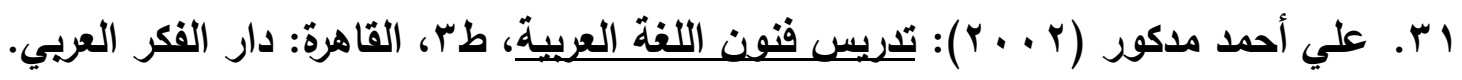

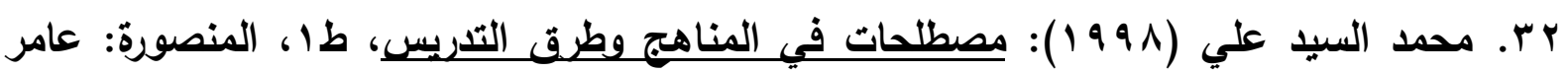

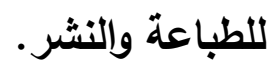

rr. ميشل تكلا جرجس ورمزي كامل حنا الله (991)): معجم المصطلحات التربوية (إنجليزيعريب)، لبنان: مكتبة لبنان ناشرون. 